\title{
Communicating Scientific Uncertainty About the COVID-19 Pandemic: Online Experimental Study of an Uncertainty-Normalizing Strategy
}

Paul K J Han ${ }^{1}$, MD, MA, MPH; Elizabeth Scharnetzki ${ }^{1}$, DPhil; Aaron M Scherer ${ }^{2}$, DPhil; Alistair Thorpe ${ }^{3}$, DPhil; Christine Lary ${ }^{1}$, DPhil; Leo B Waterston ${ }^{1}$, MA; Angela Fagerlin ${ }^{3,4}$, DPhil; Nathan F Dieckmann ${ }^{5}$, DPhil

\footnotetext{
${ }^{1}$ Center for Outcomes Research and Evaluation, Maine Medical Center Research Institute, Portland, ME, United States

${ }^{2}$ Division of General Internal Medicine, University of Iowa Carver College of Medicine, Iowa City, IA, United States

${ }^{3}$ Department of Population Health Sciences, University of Utah, Salt Lake City, UT, United States

${ }^{4}$ Salt Lake City VA Center for Informatics Decision Enhancement and Surveillance, Salt Lake City, UT, United States

${ }^{5}$ School of Nursing, Oregon Health and Science University, Portland, OR, United States
}

\section{Corresponding Author:}

Paul K J Han, MD, MA, MPH

Center for Outcomes Research and Evaluation

Maine Medical Center Research Institute

509 Forest Avenue

Portland, ME, 04101

United States

Phone: 12076617619

Email: hanp@mmc.org

\section{Abstract}

Background: Communicating scientific uncertainty about public health threats such as COVID-19 is an ethically desirable task endorsed by expert guidelines on crisis communication. However, the communication of scientific uncertainty is challenging because of its potential to promote ambiguity aversion - a well-described syndrome of negative psychological responses consisting of heightened risk perceptions, emotional distress, and decision avoidance. Communication strategies that can inform the public about scientific uncertainty while mitigating ambiguity aversion are a critical unmet need.

Objective: This study aimed to evaluate whether an "uncertainty-normalizing" communication strategy—aimed at reinforcing the expected nature of scientific uncertainty about the COVID-19 pandemic — can reduce ambiguity aversion, and to compare its effectiveness to conventional public communication strategies aimed at promoting hope and prosocial values.

Methods: In an online factorial experiment conducted from May to June 2020, a national sample of 1497 US adults read one of five versions of an informational message describing the nature, transmission, prevention, and treatment of COVID-19; the versions varied in level of expressed scientific uncertainty and supplemental focus (ie, uncertainty-normalizing, hope-promoting, and prosocial). Participants then completed measures of cognitive, emotional, and behavioral manifestations of ambiguity aversion (ie, perceived likelihood of getting COVID-19, COVID-19 worry, and intentions for COVID-19 risk-reducing behaviors and vaccination). Analyses assessed (1) the extent to which communicating uncertainty produced ambiguity-averse psychological responses; (2) the comparative effectiveness of uncertainty-normalizing, hope-promoting, and prosocial communication strategies in reducing ambiguity-averse responses; and (3) potential moderators of the effects of alternative uncertainty communication strategies.

Results: The communication of scientific uncertainty about the COVID-19 pandemic increased perceived likelihood of getting COVID-19 and worry about COVID-19, consistent with ambiguity aversion. However, it did not affect intentions for risk-reducing behaviors or vaccination. The uncertainty-normalizing strategy reduced these aversive effects of communicating scientific uncertainty, resulting in levels of both perceived likelihood of getting COVID-19 and worry about COVID-19 that did not differ from the control message that did not communicate uncertainty. In contrast, the hope-promoting and prosocial strategies did not decrease ambiguity-averse responses to scientific uncertainty. Age and political affiliation, respectively, moderated the effects of uncertainty communication strategies on intentions for COVID-19 risk-reducing behaviors and worry about COVID-19. 
Conclusions: Communicating scientific uncertainty about the COVID-19 pandemic produces ambiguity-averse cognitive and emotional, but not behavioral, responses among the general public, and an uncertainty-normalizing communication strategy reduces these responses. Normalizing uncertainty may be an effective strategy for mitigating ambiguity aversion in crisis communication efforts. More research is needed to test uncertainty-normalizing communication strategies and to elucidate the factors that moderate their effectiveness.

(J Med Internet Res 2021;23(4):e27832) doi: 10.2196/27832

\section{KEYWORDS}

uncertainty; communication; ambiguity; vaccination; COVID-19

\section{Introduction}

Public health crises such as the COVID-19 pandemic pose difficult communication challenges, due in large part to the substantial scientific uncertainty surrounding the nature and management of all new and emerging health threats [1]. This uncertainty is a defining feature of public health crises [2,3] and is important to communicate in order to foster public accountability and trust [3-5]. The communication of scientific uncertainty in public health crises is also important because it promotes more realistic expectations about the benefits of risk-reducing actions and allows people to prepare for different potential outcomes. Uncertainty communication in crisis situations has thus been a central focus of expert guidance, such as the Crisis and Emergency Risk Communication (CERC) guidelines issued by the US Centers for Disease Control and Prevention. CERC guidelines recommend that communicators both acknowledge uncertainty by clarifying what is known, what is not known, and what is being done to reduce the uncertainty, and avoid promoting excess certainty about future outcomes that cannot be controlled [6].

The challenge, however, is that uncertainty can have negative psychological effects. As CERC guidelines also acknowledge, the communication of uncertainty can heighten perceptions of risk and promote fear, panic, anxiety, emotional distress, and feelings of hopelessness and helplessness, which can prevent people from taking action [6]. These aversive psychological responses have been empirically documented by a large body of research showing that uncertainty caused by a lack of reliability, credibility, or adequacy of risk information-features of information that constitute what decision theorists have termed ambiguity [7] - produces a set of cognitive, emotional, and behavioral responses [8-10]. These include heightened risk perceptions, pessimistic appraisals of risk-reducing actions, fear and anxiety, and avoidance of decision making. These responses, collectively known as ambiguity aversion, have been demonstrated in numerous decision-making settings, including health care $[7,10,11]$.

These effects are not universal; individuals vary in their tolerance of ambiguity as well as uncertainty arising from other causes [10-12]. Furthermore, communicating uncertainty can increase, rather than decrease, individuals' confidence and trust in information when they expect such uncertainty to exist [13-15]. Nevertheless, the predominance of aversive responses to uncertainty for most individuals and situations makes the communication of scientific uncertainty in public health crises challenging [16]. Furthermore, although expert guidelines recommend adjunctive strategies, such as expressing empathy as a means of mitigating the negative psychological effects of uncertainty $[5,6]$, empirical evidence for this or other strategies is lacking, and the optimal methods for communicating uncertainty in public health crises remain unknown [2]. Consequently, available empirical evidence suggests that in these situations scientific uncertainty is rarely communicated in a clear, explicit manner, either by experts or journalists [17-19].

One promising theory-based strategy, however, may be to normalize uncertainty; that is, to emphasize that existing uncertainty is an expected experience that does not indicate an unusual deficit in people's abilities. A leading theoretical account of ambiguity aversion, the competence hypothesis, suggests that ambiguity is aversive because it lowers people's perceptions of their own competence in decision making [20]. An extension of this account, the comparative ignorance hypothesis, posits that aversion to ambiguity about a given prospect is driven by an implicit comparison with a less ambiguous prospect or the state of mind of more knowledgeable individuals [21]. Winkler has posited that ambiguity aversion may also arise from an erroneous belief in the existence of a single "true" objective probability for individual events and a discomfort with not knowing this probability [22]. Together, these theories suggest that ambiguity aversion will be heightened if decision makers' perceived competence is decreased and their comparative ignorance increased (eg, when they are made aware that relevant risk information is unavailable to them but available to others). In contrast, ambiguity aversion will be diminished if perceived competence is increased and comparative ignorance decreased. Chow and colleagues obtained experimental evidence supporting these effects by showing that individual decision makers' ambiguity aversion-manifested by their reluctance to bet on an uncertain outcome-diminished when they were made aware that the risks at hand are unknown not only to them but to all individuals; that is, they are unknowable [20,23,24].

Normalizing uncertainty as an expected state, therefore, may be a potentially effective strategy for reducing negative psychological responses to the communication of scientific uncertainty in public health crises. The overarching objective of this study was to evaluate this possibility in the real-life context of the COVID-19 pandemic. In a previous experimental study of public responses to uncertainty about a hypothetical viral pandemic [25], we found that an uncertainty-normalizing strategy did not reduce ambiguity-averse cognitive, emotional, and behavioral responses (ie, heightened risk perceptions and worry and diminished vaccination intentions) to scientific 
uncertainty about the pandemic. However, the generalizability of these findings was limited by the hypothetical nature of the study. In the current study, we addressed this limitation by evaluating the effects of an uncertainty-normalizing strategy in a real public health crisis. Its specific objective was to test whether normalizing uncertainty reduces ambiguity-averse responses, compared to commonly used strategies aimed at promoting either (1) hope or (2) prosocial values [26-28]. These alternative strategies have been broadly implemented in public information campaigns about COVID-19, and focus on mitigating hopelessness, helplessness, and stigmatization-important adverse responses to public health crises $[3,5,6]$. However, because these strategies do not directly target perceptions of, or responses to, uncertainty, they should be less effective in reducing ambiguity aversion than an uncertainty-normalizing strategy.

To evaluate this possibility, we conducted an online survey-based experiment comparing alternative approaches to communicating scientific uncertainty about multiple aspects of the COVID-19 pandemic, including its controllability, prognosis, and severity. The experiment tested the following hypotheses:

- Hypothesis 1 (H1). The communication of uncertainty about the COVID-19 pandemic will result in ambiguity-averse psychological responses-consisting of greater perceived likelihood of developing COVID-19, greater worry about COVID-19, and lower intentions for COVID-19 risk-reducing behaviors-compared to the noncommunication of uncertainty.

- Hypothesis $2(\mathrm{H} 2)$. Ambiguity-averse responses to the communication of uncertainty about the COVID-19 pandemic will be reduced by uncertainty-normalizing language but not by either hope-promoting or prosocial language.

As an exploratory objective, we also evaluated the extent to which individual differences, including sociodemographic characteristics (ie, age, gender, and education), political affiliation, health literacy, trait-level risk aversion, trait-level ambiguity aversion, and dispositional optimism—all factors that might influence people's responses to medical uncertainty $[11,12,25,29,30]$ - might moderate the effects of these different uncertainty communication strategies.

\section{Methods}

\section{Study Design and Experimental Manipulation}

The study was part of a larger online experiment, hosted by the internet survey vendor Qualtrics, designed to test different strategies, including language aimed at promoting hope and prosocial values, for communicating to the general public about the nature and prevention of COVID-19. This study focused specifically on strategies for communicating about uncertainty surrounding the COVID-19 pandemic. All alternative strategies were created by adding language to basic information on the nature, transmission, prevention, and treatment of COVID-19, reproduced from a public website produced by a government public health department [31]. This basic information contained no explicit communication of scientific uncertainty and served as the control strategy. Supplementing this basic information with additional language resulted in a total of five alternative uncertainty communication strategies, which constituted separate experimental conditions to which participants were randomly assigned: (1) control, (2) uncertainty, (3) uncertainty + uncertainty-normalizing, (4) uncertainty + hope-promoting, and (5) uncertainty + prosocial.

The uncertainty condition highlighted the existence of scientific uncertainty about the controllability, prognosis, and severity of the COVID-19 pandemic. The uncertainty + uncertainty-normalizing condition combined expressed uncertainty with language emphasizing the unknowability of these various aspects of COVID-19 and the expected nature of scientific uncertainty. The uncertainty + hope-promoting condition combined expressed uncertainty with language conveying optimism about future advances in knowledge and control over the pandemic. The uncertainty + prosocial condition combined expressed uncertainty with language encouraging awareness of obligations to other community members and concern for the collective good. The alternative uncertainty communication strategies varied in length from 940 to 1273 words; the full text of all strategies is presented in Multimedia Appendix 1.

\section{Study Population and Recruitment}

The study population consisted of a national sample of adult members (aged $\geq 18$ years) of the US public belonging to a voluntary opt-in web survey panel professionally managed by the internet survey vendor Qualtrics. Panel members have experience and interest in completing online surveys for marketing purposes, for which they are provided modest monetary incentives. Qualtrics maintains sociodemographic and geographic data on panel members, which provides the capacity to target recruitment to prespecified quotas in order to achieve a sociodemographically diverse study sample. This study employed quotas aimed at obtaining a balanced distribution by age, gender, race, geographic region of the United States, education level (ie, $\geq 20 \%$ high school diploma or less), and income (ie, $\geq 50 \%$ annual income of US $\$ 50,000$ or less), and to exclude participants who reported a current or prior diagnosis of COVID-19. To ensure data quality, we excluded participants who gave logically inconsistent responses to two screener questions about participants' attitudes toward risk-reducing behaviors or whose survey completion time was below 12 minutes - the time cut point accounting for the majority of inconsistent responses in preliminary fielding of the study.

The study was approved by the MaineHealth Institutional Review Board. The survey was fielded from May 7 to June 11, 2020; during this time, the number of total coronavirus infections in the United States increased from $>1.2$ million to $>1.6$ million, and total deaths increased from $>77,200$ to $>98,000$ [32].

\section{Measures}

After reading their randomly assigned informational vignettes, participants completed a survey questionnaire consisting of the measures summarized below. 


\section{Outcome Variables}

Perceived uncertainty about COVID-19 served as the manipulation check for the study and was assessed using a 6 -item scale ( $\alpha=.71)$ developed for this study (see Multimedia Appendix 2). This measure assessed participants' perceptions of uncertainty arising from various sources (ie, probability, ambiguity, and complexity) and pertaining to various issues (ie, controllability, prognosis, and severity of the COVID-19 pandemic) raised in the experimental vignettes. Example items include "There are conflicting estimates of how long the COVID-19 pandemic will past." Likert scale response options ranged from 1 (strongly disagree) to 7 (strongly agree).

Perceived likelihood of getting COVID-19 was assessed with a single item used in prior studies [25,33,34]: "How likely does it feel that you will get COVID-19 within the next month?" Likert scale response options ranged from 1 (not at all) to 7 (very).

Worry about COVID-19 was assessed with a single item used in prior studies [25,33,34]: "How worried are you about getting COVID-19 within the next month?" Likert scale response options ranged from 1 (not at all) to 7 (very).

Intentions for COVID-19 risk-reducing behaviors was assessed by measuring participants' willingness to follow 14 recommended COVID-19 risk-reducing behaviors (eg, handwashing, avoiding social gatherings, and wearing masks) (see Multimedia Appendix 2). Likert scale response options ranged from 0 (I am not planning to follow this guideline at all) to 100 (I am planning to follow this guideline fully). Participants' responses were averaged to create a composite score $(\alpha=.95)$.

Intentions for vaccination was assessed with a single item used in prior studies [25,33,34]: "If a vaccine becomes available for COVID-19, how likely would you be to get vaccinated against COVID-19?" Likert scale response options ranged from 1 (definitely would not get vaccinated) to 7 (definitely would get vaccinated).

\section{Covariates and Potential Moderators}

Sociodemographic characteristics included age (ie, <30, 30-39, $40-49,50-59,60-69$, and $\geq 70$ years), gender, race, and political affiliation (ie, Democrat, independent or other, and Republican).
Subjective health literacy was assessed using an abbreviated, single-item version of a validated health literacy screening measure [35]: "How often do you have someone (like a family member, friend, hospital/clinic worker, or caregiver) help you read instructions, pamphlets, or other written health materials from your doctor or pharmacy?" Likert scale response options ranged from 1 (never) to 5 (always).

\section{Data Analysis}

To compare the effectiveness of alternative uncertainty communication strategies in reducing cognitive, emotional, and behavioral manifestations of ambiguity aversion, we fit analysis of variance models with perceived likelihood of getting COVID-19, worry about COVID-19, and intentions for both risk-reducing behaviors and vaccination as dependent variables and communication strategy as the independent variable. For each dependent variable, we used prespecified contrasts to assess the following: (1) the extent to which the communication of uncertainty produced ambiguity-averse responses, compared to the noncommunication of uncertainty (H1), and (2) the extent to which the three alternative uncertainty communication strategies (ie, uncertainty-normalizing, hope-promoting, prosocial) reduced ambiguity-averse responses (H2) (see Figure $1)$.

H1 was assessed by the contrast between the uncertainty and control conditions, while $\mathrm{H} 2$ was assessed by contrasts between each of the alternative uncertainty communication strategies and both the uncertainty and control conditions. For each contrast, we estimated the effect size by calculating Cohen $d$, which represents the standardized mean difference between two groups [36].

To explore potential moderating effects of sociodemographic characteristics (ie, age, gender, race, and political affiliation) and subjective health literacy, we fit separate models with perceived risk of COVID-19, worry about COVID-19, and intentions for both risk-reducing behaviors and vaccination as dependent variables and communication strategy as the independent variable; we entered relevant interaction terms one at a time. All analyses were conducted using SPSS, version 27.0 (IBM Corp). 
Figure 1. Study design. Alternative uncertainty communication strategies and between-group comparisons. H1: Hypothesis 1; H2: Hypothesis 2 .

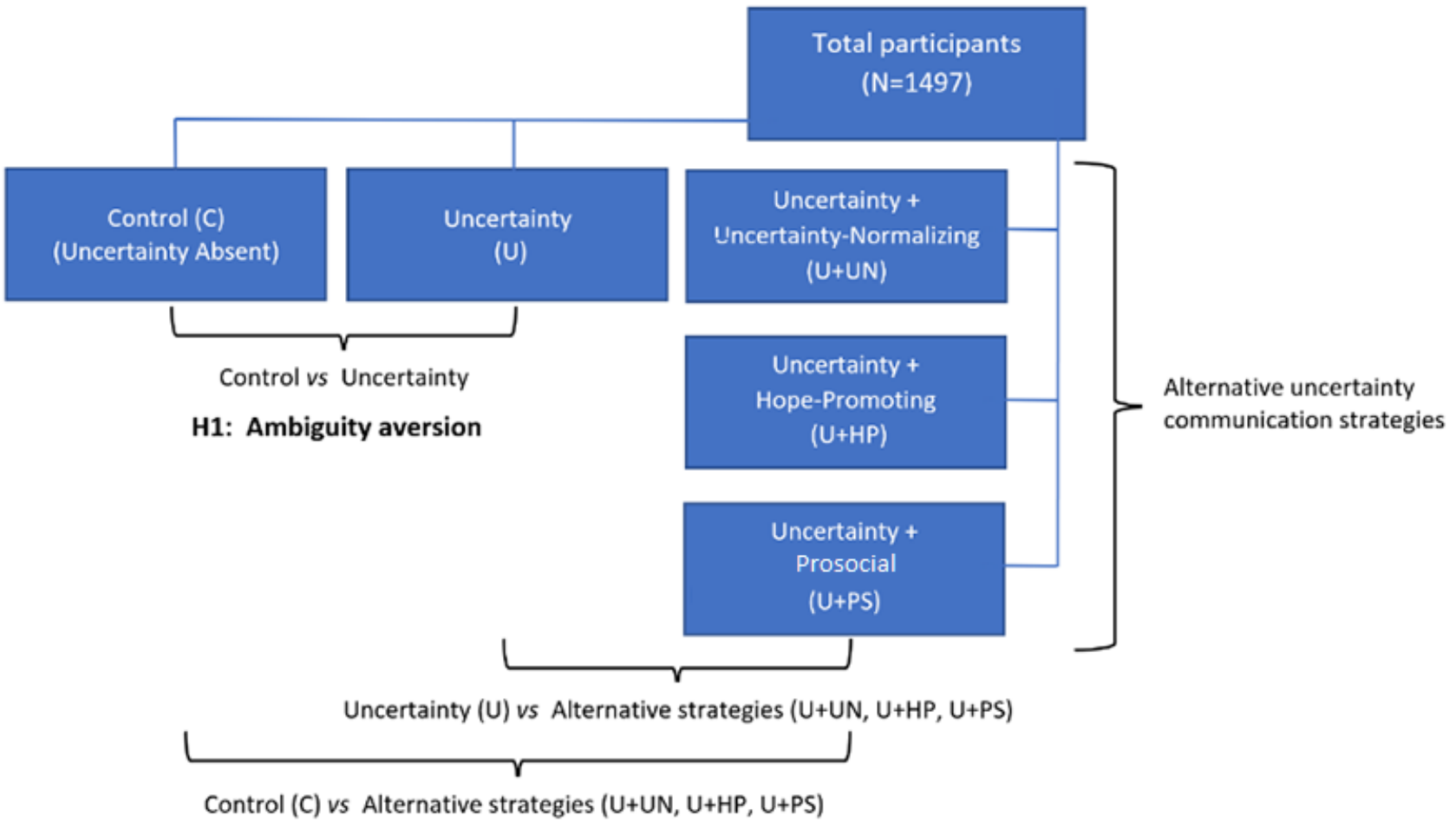

H2: Reduction of ambiguity aversion

\section{Results}

\section{Overview}

In our primary conditions, we received data from 1524 respondents. We excluded 2 respondents who gave inconsistent responses and another 25 individuals who reported current or previous COVID-19 illness, leaving a final sample of 1497 respondents (see Table 1). Data were assumed to be missing at random; thus, we utilized a listwise deletion strategy for participants with missing data on any of the outcome measures.

On average, participants took 27.82 (SD 34.59) minutes to complete the study. There were no significant between-group differences in time of completion of the experimental task $\left(F_{7,2386}=0.479 ; P=.85\right)$, suggesting that the cognitive effort required by the task was similar across conditions. 
Table 1. Sample population characteristics.

\begin{tabular}{|c|c|}
\hline Characteristic & Value $(\mathrm{N}=1497), \mathrm{n}(\%)$ \\
\hline \multicolumn{2}{|l|}{ Age (years) } \\
\hline$<30$ & $306(20.4)$ \\
\hline $30-39$ & $229(15.3)$ \\
\hline $40-49$ & $209(14.0)$ \\
\hline $50-59$ & $183(12.2)$ \\
\hline $60-69$ & $227(15.2)$ \\
\hline$\geq 70$ & 343 (22.9) \\
\hline \multicolumn{2}{|l|}{ Gender } \\
\hline Male & $743(49.6)$ \\
\hline Female & $748(50.0)$ \\
\hline Other or prefer not to say & $6(0.4)$ \\
\hline \multicolumn{2}{|l|}{ Race } \\
\hline White & $1003(67.0)$ \\
\hline Black or African American & $178(11.9)$ \\
\hline Asian & $147(9.8)$ \\
\hline Multiracial or other & $169(11.3)$ \\
\hline \multicolumn{2}{|l|}{ Education } \\
\hline Less than high school & $264(17.6)$ \\
\hline High school graduate & $242(16.2)$ \\
\hline Some college or trade school & $396(26.5)$ \\
\hline College graduate or higher & $595(39.7)$ \\
\hline \multicolumn{2}{|l|}{ Income (US \$) } \\
\hline $0-24,999$ & $394(26.3)$ \\
\hline $25,000-49,999$ & $371(24.8)$ \\
\hline $50,000-99,999$ & $370(24.7)$ \\
\hline $100,000-149,999$ & $235(15.7)$ \\
\hline$\geq 150,000$ & $127(8.5)$ \\
\hline \multicolumn{2}{|l|}{ Political affiliation } \\
\hline Democrat & $540(36.1)$ \\
\hline Republican & $438(29.3)$ \\
\hline Independent or other ${ }^{\mathrm{a}}$ & $519(34.7)$ \\
\hline
\end{tabular}

${ }^{\mathrm{a}}$ Includes other third party or no party affiliation.

\section{Manipulation Check}

Supporting the intended effect of the experimental manipulation, perceived uncertainty about COVID-19 was significantly higher in all experimental conditions containing uncertainty $\left(F_{4,1492}=3.52 ; \eta^{2}=0.009 ; P=.007\right)$ - that is, uncertainty $(d=-0.28$; $P=.001)$, uncertainty + uncertainty-normalizing ( $d=-0.23$; $P=.006)$, uncertainty + hope-promoting $(d=-0.20 ; P=.01)$, and uncertainty + prosocial $(d=-0.22 ; P=.007)$ — compared to the control condition containing no uncertainty. These differences were in the small effect-size range.

\section{Perceived Likelihood of Getting COVID-19}

Consistent with an ambiguity-averse cognitive response to the communication of uncertainty (H1), perceived likelihood of getting COVID-19 was significantly higher in the uncertainty condition than in the control condition $\left(F_{4,1492}=2.95 ; \eta^{2}=0.008\right.$; $P=.02$ ) (see Figure 2A). Supporting the effectiveness of the uncertainty-normalizing strategy in reducing ambiguity aversion (H2), perceived likelihood of getting COVID-19 was not significantly different for the uncertainty + uncertainty-normalizing condition compared to the control condition $(d=-0.04 ; \quad P=.66)$. However, this ambiguity aversion-reducing effect was not seen for the hope-promoting 
$(d=-0.18 ; \quad P=.03) \quad$ or $\quad$ prosocial $\quad(d=-0.23 ; \quad P=.005)$ communication strategies; perceived likelihood remained significantly higher for these conditions than for the control condition.

Figure 2. Effects of uncertainty and uncertainty communication strategies on cognitive, emotional, and behavioral manifestations of ambiguity aversion. Asterisks indicate statistically significant pairwise differences $(P<.05)$; error bars indicate standard error. U: uncertainty; U+HP: uncertainty + hope-promoting; U+PS: uncertainty + prosocial; U+UN: uncertainty + uncertainty-normalizing.

\section{A. Perceived likelihood of COVID-19}

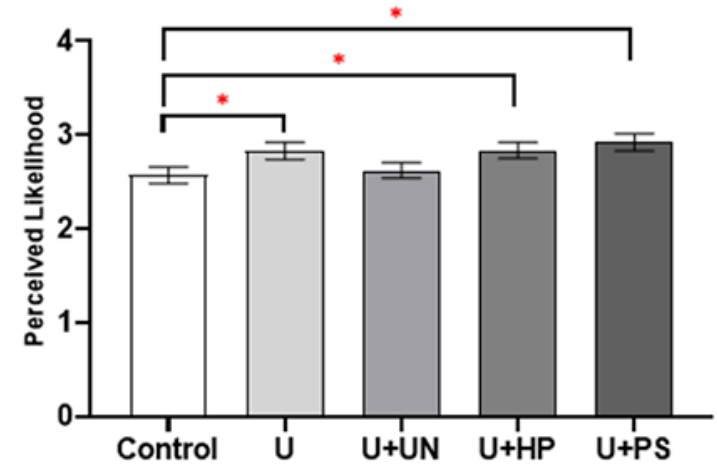

C. Intentions for risk-reducing behaviors

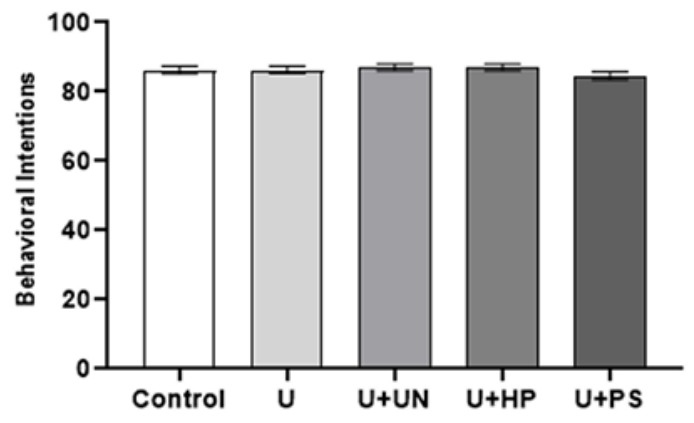

B. Worry about COVID-19

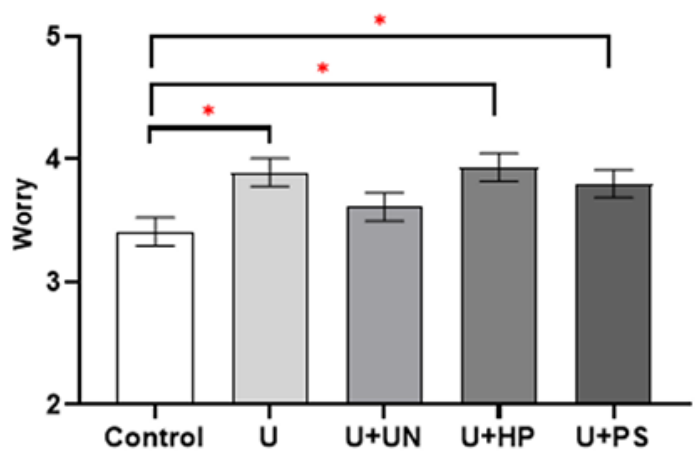

D. Intentions for COVID-19 vaccination

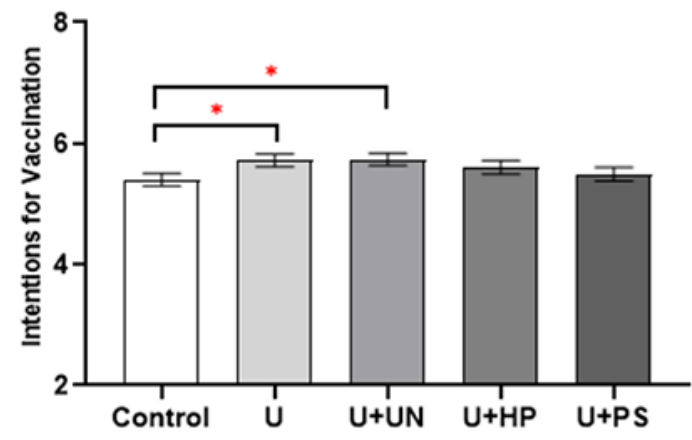

\section{Worry About COVID-19}

Consistent with an ambiguity-averse emotional response to the communication of uncertainty (H1), worry about COVID-19 was significantly higher in the uncertainty condition than in the control condition $\left(F_{4,1492}=3.65 ; \eta^{2}=0.01 ; P=.006\right)$ (see Figure 2B). Supporting the effectiveness of the uncertainty-normalizing strategy in reducing ambiguity aversion (H2), worry was not significantly different for the uncertainty + uncertainty-normalizing condition compared to the control condition $(d=-0.10 ; P=.21)$. However, this ambiguity aversion-reducing effect was not seen for the hope-promoting $(d=-0.27 ; \quad P=.001) \quad$ or prosocial $\quad(d=-0.20 ; \quad P=.02)$ communication strategies; worry remained significantly higher for these conditions than for the control condition.

\section{Intentions for COVID-19 Risk-Reducing Behaviors and Vaccination}

Inconsistent with an ambiguity-averse behavioral response to uncertainty, intentions regarding COVID-19 risk-reducing behaviors $\left(\eta^{2}=0.002 ; P=.49\right)$ and vaccination $\left(\eta^{2}=0.005 ; P=.14\right)$ showed no significant differences between any of the experimental conditions (see Figure 2C and D). However, prespecified contrasts revealed higher vaccination intentions in both the uncertainty $(d=-0.17 ; P=.04)$ and the uncertainty + uncertainty-normalizing $(d=-0.18 ; P=.03)$ conditions compared to the control condition, suggesting that the communication of uncertainty itself motivated vaccination intentions and that the addition of uncertainty-normalizing language preserved this motivation (see Figure 2D).

\section{Moderating Effects}

Two factors, age and political affiliation, were found to moderate the effects of uncertainty communication strategy on different ambiguity-averse responses to the communication of uncertainty. Age moderated the effect of communication strategy on intentions for COVID-19 risk-reducing behaviors $\left(F_{20,1445}=1.86\right.$; $\eta \rho^{2}=0.025 ; P=.01$ ), such that older participants (ie, aged 50 years and older) generally reported higher intentions in all of the supplementary uncertainty communication conditions compared to the control condition, while younger participants (ie, less than 50 years of age) generally reported lower intentions (see Figure 3, top plot).

Political affiliation showed a weaker interaction with uncertainty communication strategy on worry about COVID-19 $\left(\eta \rho^{2}=0.010\right.$; $P=.06$ ), such that self-reported Republicans had lower worry in 
the uncertainty + uncertainty-normalizing condition compared to the control condition, while self-reported Democrats and independents had higher worry (see Figure 3, bottom plot). In other words, the uncertainty-normalizing strategy reduced ambiguity aversion to a greater extent for Republicans than for
Democrats. Democrats also had higher worry in the prosocial condition compared to the control condition, while Republicans and independents had lower worry.

No significant moderating effects were noted for other sociodemographic factors or subjective health literacy.

Figure 3. Moderators of the effects of uncertainty communication strategy on manifestations of ambiguity aversion: age and political affiliation. U: uncertainty; U+HP: uncertainty + hope-promoting; U+PS: uncertainty + prosocial; U+UN: uncertainty + uncertainty-normalizing.
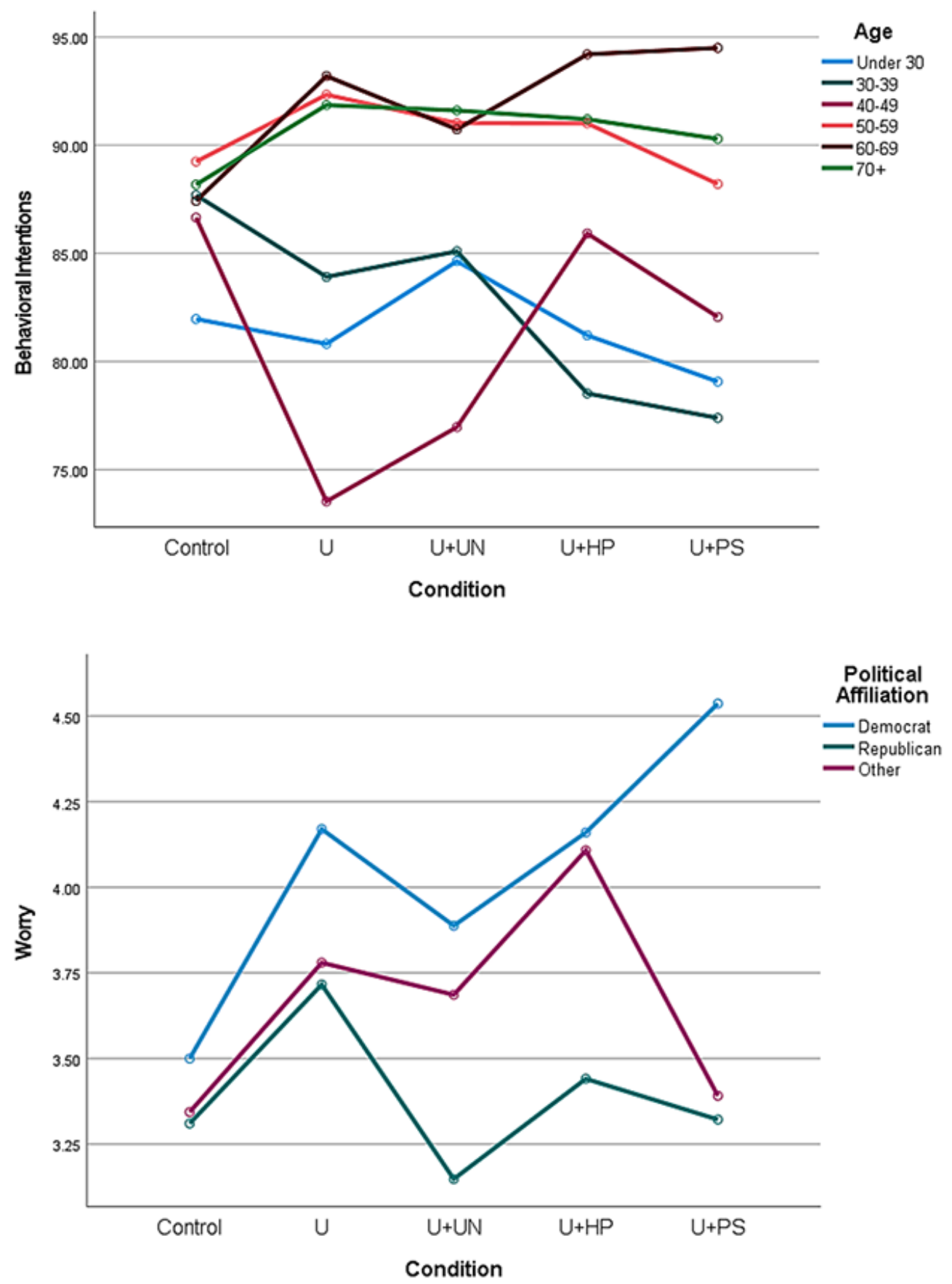

\section{Discussion}

This experimental study evaluated the comparative effectiveness of different communication strategies in reducing ambiguity-averse cognitive, emotional, and behavioral responses to uncertainty in information about the COVID-19 pandemic. We believe its findings have several implications for future efforts to understand and improve the communication of uncertainty in public health crises. 
Consistent with predictions, a strategy aimed at normalizing uncertainty as an expected state of affairs was effective in reducing at least some aversive psychological responses to the communication of uncertainty, whereas widely used alternative strategies aimed at promoting hope and prosocial values had no such effect. A major barrier to open, explicit communication of the uncertainties that inevitably exist during public health crises is a real concern about exacerbating both the perception of vulnerability as well as feelings of fear and panic among the general public $[2,3,5,6]$. Our findings suggest, however, that language aimed at normalizing these uncertainties can reduce aversive cognitive and emotional responses to them. When uncertainty-normalizing language was added to a message that communicated scientific uncertainty about the COVID-19 pandemic, levels of COVID-19 risk perceptions and worry did not differ from those produced by a message that did not communicate scientific uncertainty. In other words, uncertainty-normalizing language neutralized ambiguity aversion. The overall size of this effect was relatively small, and although uncertainty-normalizing language resulted in lower levels of COVID-19 risk perceptions and worry than those produced by a message that communicated uncertainty alone, this difference was not statistically significant. Nevertheless, even small effects in reducing aversive psychological responses to uncertainty may be beneficial in large-scale efforts to communicate with the general public about health crises. If our findings can be replicated and validated, they suggest a promising new approach to inoculating people against the vulnerability and fear that typically accompany the communication of uncertainty in these situations.

Contrary to predictions, uncertainty-normalizing language had no effect on intentions for COVID-19 risk-reducing behaviors or vaccination. Notably, however, the communication of uncertainty itself also had no effect; it neither decreased nor increased behavioral intentions. In other words, ambiguity aversion in this study was manifest cognitively and emotionally, but not behaviorally. This pattern may be attributable to several factors. Potential negative effects of scientific uncertainty about COVID-19 on intentions for risk-reducing behaviors may have been attenuated by the legally mandated nature of several of these behaviors (eg, mandatory quarantines and regulations requiring social distancing and use of masks). Furthermore, uncertainties about the controllability, prognosis, and severity of the COVID-19 pandemic may have mixed, opposing effects on behavioral intentions. They may decrease intentions by fostering skepticism about the benefits of risk-reducing behaviors, thereby promoting a tendency toward inaction, which is consistent with ambiguity aversion. At the same time, these different uncertainties may also increase intentions by promoting fear about the consequences of avoiding risk-reducing behaviors, thereby promoting a tendency toward action, which is consistent with ambiguity tolerance. More research is needed to understand the factors that moderate people's behavioral responses to different uncertainties and favor either inaction or action.

Our study sheds light on at least some of these factors. Age moderated the effect of communication strategy on intentions for COVID-19 risk-reducing behaviors. For adults over 50 years of age, all active uncertainty communication strategies resulted in higher behavioral intentions compared to the control (ie, no uncertainty) strategy, while for younger adults, all active uncertainty communication strategies resulted in lower intentions. This moderating effect may be attributable to several factors. Older adults have been identified as being at higher risk for complications of COVID-19 and may, thus, be more motivated to take action in the face of uncertainty. Political affiliation also appeared to partially moderate the effect of communication strategy on worry about COVID-19; the uncertainty-normalizing strategy was more effective in reducing ambiguity aversion for Republicans than for Democrats. This moderating effect is intriguing, and its causes are unclear. Political party affiliation has been shown to influence COVID-19 risk perceptions and intentions for risk-reducing behaviors; Republicans generally demonstrate lower risk perceptions and behavioral intentions than Democrats [37-39]. The differential worry-reducing effect of uncertainty normalization for Republicans versus Democrats suggests the existence of respectively opposing propensities toward either minimizing feelings of vulnerability in the face of uncertainty (for Republicans) or else maximizing them (for Democrats). Political affiliation may be a proxy for numerous factors, including ideologies, values, and worldviews, that may predispose people to more optimistic or pessimistic appraisals of uncertain threats [40]. More research is needed to elucidate how these and other factors produce differential responses to different uncertainty communication strategies and to identify other important moderators.

Our study had several limitations that qualify its findings. The sample consisted of web survey panel members who, by virtue of their willingness to participate regularly in market research and other studies, may not be representative of the general population. However, the sample was large and both geographically and sociodemographically diverse, providing support for the external validity of its findings. Nevertheless, more research is needed to assess the reproducibility of our findings and their generalizability to other populations. The alternative uncertainty communication strategies tested in this study varied modestly in length; however, the absence of significant between-group differences in study completion time suggests that our findings are not attributable solely to differences in cognitive burden or effort. The uncertainty-normalizing language tested in this study was also novel and unvalidated, and it may have contained unintended hope-promoting or prosocial messages. We believe the significant between-group differences observed in our study argue against this possibility; however, further research is needed to ascertain the precision and efficacy of our uncertainty-normalizing language in conveying the normal, expected nature of uncertainty. We also conducted multiple exploratory analyses to identify potential moderators of the effects of different communication strategies; the two significant interactions identified could thus have resulted from chance, and further research is needed to confirm these findings. Finally, some key constructs (ie, perceived uncertainty about COVID-19 and intentions for COVID-19 risk-reducing behaviors) were assessed using new measures that have yet to be validated. Other constructs were assessed using existing single-item measures; 
however, similar measures have been used in prior studies and shown to have predictive validity [25,30,41-43].

In spite of these limitations, this study yields important new insights on the nature and extent of aversion to ambiguity in the context of the COVID-19 pandemic and on a new and potentially effective uncertainty communication strategy that can minimize this aversion. It remains for future research to confirm our findings and to develop more effective strategies for communicating the unavoidable and irreducible scientific uncertainties that complicate all public health crises.

\section{Acknowledgments}

This work was supported by a Pilot Project Program Award to PH from the Northern New England Clinical \& Translational Research Network (1U54GM115516-01). The funding agreement ensured the authors' independence in designing the study, interpreting the data, and in writing and publishing the report.

\section{Authors' Contributions}

PH conceived and designed the study, developed the experimental conditions and measures, conducted the experiments, analyzed the data, and drafted and revised the manuscript. ES, AS, AT, CL, LW, AF, and ND contributed to the development of the experimental conditions and measures, provided input on data analysis, and revised the manuscript. ES also programmed the survey and analyzed the data. All authors approved the final version.

\section{Conflicts of Interest}

None declared.

\section{Multimedia Appendix 1}

Experimental conditions.

[DOCX File, $30 \mathrm{~KB}$-Multimedia Appendix 1]

\section{Multimedia Appendix 2}

Measures developed in this study.

[DOCX File, 16 KB-Multimedia Appendix 2]

\section{References}

1. Freimuth VS. Order out of chaos: The self-organization of communication following the anthrax attacks. Health Commun 2006;20(2):141-148. [doi: 10.1207/s15327027hc2002 5] [Medline: 16965251]

2. Liu BF, Bartz L, Duke N. Communicating crisis uncertainty: A review of the knowledge gaps. Public Relat Rev 2016 Sep;42(3):479-487. [doi: 10.1016/j.pubrev.2016.03.003]

3. Seeger MW. Best practices in crisis communication: An expert panel process. J Appl Commun Res 2006 Aug;34(3):232-244. [doi: 10.1080/00909880600769944]

4. Berg JW. All for one and one for all: Informed consent and public health. Houst Law Rev 2012;50(1):1-40 [FREE Full $\underline{\text { text] }}$

5. Covello VT. Best practices in public health risk and crisis communication. J Health Commun 2003;8 Suppl 1:5-8; discussion 148. [doi: 10.1080/713851971] [Medline: $\underline{14692565]}$

6. Crisis and Emergency Risk Communication (CERC) manual. US Centers for Disease Control and Prevention. 2019. URL: https://emergency.cdc.gov/cerc/manual/index.asp [accessed 2021-04-05]

7. Ellsberg D. Risk, ambiguity, and the savage axioms. Q J Econ 1961 Nov;75(4):643. [doi: 10.2307/1884324]

8. Machina MJ, Siniscalchi M. Ambiguity and ambiguity aversion. In: Machina MJ, Viscusi WK, editors. Handbook of the Economics of Risk and Uncertainty. Oxford, UK: North-Holland; 2014:729-807.

9. Golman R, Gurney N, Loewenstein G. Information gaps for risk and ambiguity. Psychol Rev 2021 Jan;128(1):86-103. [doi: 10.1037/rev0000252] [Medline: 32757571]

10. Camerer C, Weber M. Recent developments in modeling preferences: Uncertainty and ambiguity. J Risk Uncertain 1992 Oct;5(4):325-370. [doi: 10.1007/bf00122575]

11. Han PKJ, Reeve BB, Moser RP, Klein WMP. Aversion to ambiguity regarding medical tests and treatments: Measurement, prevalence, and relationship to sociodemographic factors. J Health Commun 2009 Sep;14(6):556-572 [FREE Full text] [doi: 10.1080/10810730903089630] [Medline: 19731127]

12. Hillen MA, Gutheil CM, Strout TD, Smets EM, Han PK. Tolerance of uncertainty: Conceptual analysis, integrative model, and implications for healthcare. Soc Sci Med 2017 May;180:62-75 [FREE Full text] [doi: 10.1016/j.socscimed.2017.03.024] [Medline: 28324792]

13. Du N, Budescu DV, Shelly MK, Omer TC. The appeal of vague financial forecasts. Organ Behav Hum Decis Process 2011 Mar;114(2):179-189. [doi: 10.1016/j.obhdp.2010.10.005] 
14. Morss R, Demuth J, Lazo J. Communicating uncertainty in weather forecasts: A survey of the US public. Weather Forecast 2008;23(5):974-991. [doi: 10.1175/2008waf2007088.1]

15. Wegwarth O, Wagner G, Spies C, Hertwig R. Assessment of German public attitudes toward health communications with varying degrees of scientific uncertainty regarding COVID-19. JAMA Netw Open 2020 Dec 01;3(12):e2032335 [FREE Full text] [doi: 10.1001/jamanetworkopen.2020.32335] [Medline: 33301021]

16. Frewer L. The public and effective risk communication. Toxicol Lett 2004 Apr 01;149(1-3):391-397. [doi: 10.1016/j.toxlet.2003.12.049] [Medline: 15093286]

17. Goodall C, Sabo J, Cline R, Egbert N. Threat, efficacy, and uncertainty in the first 5 months of national print and electronic news coverage of the H1N1 virus. J Health Commun 2012;17(3):338-355. [doi: 10.1080/10810730.2011.626499] [Medline: 22188164]

18. Singer E. A question of accuracy: How journalists and scientists report research on hazards. J Commun 1990 Dec;40(4):102-116 [FREE Full text] [doi: 10.1111/j.1460-2466.1990.tb02284.x]

19. Pellechia MG. Trends in science coverage: A content analysis of three US newspapers. Public Underst Sci 2016 Dec 21;6(1):49-68. [doi: 10.1088/0963-6625/6/1/004]

20. Heath C, Tversky A. Preference and belief: Ambiguity and competence in choice under uncertainty. J Risk Uncertain 1991 Jan;4(1):5-28. [doi: 10.1007/BF00057884]

21. Fox CR, Tversky A. Ambiguity aversion and comparative ignorance. Q J Econ 1995 Aug;110(3):585-603 [FREE Full text] [doi: 10.2307/2946693]

22. Winkler RL. Ambiguity, probability, preference, and decision analysis. J Risk Uncertain 1991 Jul;4(3):285-297. [doi: 10.1007/BF00114158]

23. Chow CC, Sarin RK. Comparative ignorance and the Ellsberg Paradox. J Risk Uncertain 2001;22:129-139. [doi: 10.1023/A:1011157509006]

24. Chow CC, Sarin RK. Known, unknown, and unknowable uncertainties. Theory Decis 2002;52:127-138. [doi: 10.1023/A:1015544715608]

25. Han P, Zikmund-Fisher B, Duarte C, Knaus M, Black A, Scherer A, et al. Communication of scientific uncertainty about a novel pandemic health threat: Ambiguity aversion and its mechanisms. J Health Commun 2018;23(5):435-444 [FREE Full text] [doi: 10.1080/10810730.2018.1461961] [Medline: 29648962]

26. Keshavarz S, Coventry K, Fleming P. Relative deprivation and hope: Predictors of risk behavior. J Gambl Stud 2020 Dec 16:1 [FREE Full text] [doi: 10.1007/s10899-020-09989-4] [Medline: 33326060]

27. Heffner J, Vives M, FeldmanHall O. Emotional responses to prosocial messages increase willingness to self-isolate during the COVID-19 pandemic. Pers Individ Dif 2021 Feb 15;170:110420 [FREE Full text] [doi: 10.1016/j.paid.2020.110420] [Medline: 33082614$]$

28. Jung H, Albarracín D. Concerns for others increases the likelihood of vaccination against influenza and COVID-19 more in sparsely rather than densely populated areas. Proc Natl Acad Sci U S A 2021 Jan 05;118(1):1-8 [FREE Full text] [doi: 10.1073/pnas.2007538118] [Medline: 33443183]

29. Strout T, Hillen M, Gutheil C, Anderson E, Hutchinson R, Ward H, et al. Tolerance of uncertainty: A systematic review of health and healthcare-related outcomes. Patient Educ Couns 2018 Sep;101(9):1518-1537. [doi: 10.1016/j.pec.2018.03.030] [Medline: 29655876]

30. Han P, Klein W, Lehman T, Killam B, Massett H, Freedman A. Communication of uncertainty regarding individualized cancer risk estimates: Effects and influential factors. Med Decis Making 2011;31(2):354-366 [FREE Full text] [doi: 10.1177/0272989X10371830] [Medline: 20671211]

31. COVID-19: General information. Maine Center for Disease Control \& Prevention. 2020. URL: https://www.maine.gov/ dhhs/mecdc/infectious-disease/epi/airborne/coronavirus/general-information.shtml [accessed 2020-07-01]

32. Coronavirus world map: Tracking the global outbreak. The New York Times. 2020. URL: https://www.nytimes.com/ interactive/2020/world/coronavirus-maps.html [accessed 2020-06-01]

33. Zikmund-Fisher BJ, Scherer AM, Knaus M, Das E, Fagerlin A. Discussion of average versus extreme case severity in pandemic risk communications. Emerg Infect Dis 2017 Apr;23(4):706-708 [FREE Full text] [doi: 10.3201/eid2304.161600] [Medline: 28322691]

34. Fagerlin A, Valley TS, Scherer AM, Knaus M, Das E, Zikmund-Fisher BJ. Communicating infectious disease prevalence through graphics: Results from an international survey. Vaccine 2017 Jul 13;35(32):4041-4047 [FREE Full text] [doi: 10.1016/j.vaccine.2017.05.048] [Medline: 28647168]

35. Chew LD, Griffin JM, Partin MR, Noorbaloochi S, Grill JP, Snyder A, et al. Validation of screening questions for limited health literacy in a large VA outpatient population. J Gen Intern Med 2008 May;23(5):561-566 [FREE Full text] [doi: 10.1007/s11606-008-0520-5] [Medline: 18335281]

36. Cohen J. Statistical Power Analysis for the Behavioral Sciences. 2nd edition. Mahwah, NJ: Lawrence Erlbaum Associates; 1988.

37. Bruine de Bruin W, Saw H, Goldman D. Political polarization in US residents' COVID-19 risk perceptions, policy preferences, and protective behaviors. J Risk Uncertain 2020 Nov 18:1-18 [FREE Full text] [doi: 10.1007/s11166-020-09336-3] [Medline: $\underline{33223612]}$ 
38. van Holm EJ, Monaghan J, Shahar DC, Messina J, Surprenant C. The impact of political ideology on concern and behavior during COVID-19. SSRN 2020 Apr:1. [doi: 10.2139/ssrn.3573224]

39. Kushner Gadarian S, Goodman SW, Pepinsky TB. Partisanship, health behavior, and policy attitudes in the early stages of the COVID-19 pandemic. SSRN 2020 Mar:1. [doi: 10.2139/ssrn.3562796]

40. Jost JT. Ideological asymmetries and the essence of political psychology. Polit Psychol 2017 Mar 15;38(2):167-208. [doi: 10.1111/pops.12407]

41. Diefenbach M, Miller S, Daly M. Specific worry about breast cancer predicts mammography use in women at risk for breast and ovarian cancer. Health Psychol 1999 Sep;18(5):532-536. [doi: 10.1037//0278-6133.18.5.532] [Medline: 10519469]

42. Lipkus I, Iden D, Terrenoire J, Feaganes J. Relationships among breast cancer concern, risk perceptions, and interest in genetic testing for breast cancer susceptibility among African-American women with and without a family history of breast cancer. Cancer Epidemiol Biomarkers Prev 1999 Jun;8(6):533-539 [FREE Full text] [Medline: 10385144]

43. Han P, Klein W, Killam B, Lehman T, Massett H, Freedman A. Representing randomness in the communication of individualized cancer risk estimates: Effects on cancer risk perceptions, worry, and subjective uncertainty about risk. Patient Educ Couns 2012 Jan;86(1):106-113 [FREE Full text] [doi: 10.1016/j.pec.2011.01.033] [Medline: 21377311]

\author{
Abbreviations \\ CERC: Crisis and Emergency Risk Communication \\ H1: Hypothesis 1 \\ H2: Hypothesis 2
}

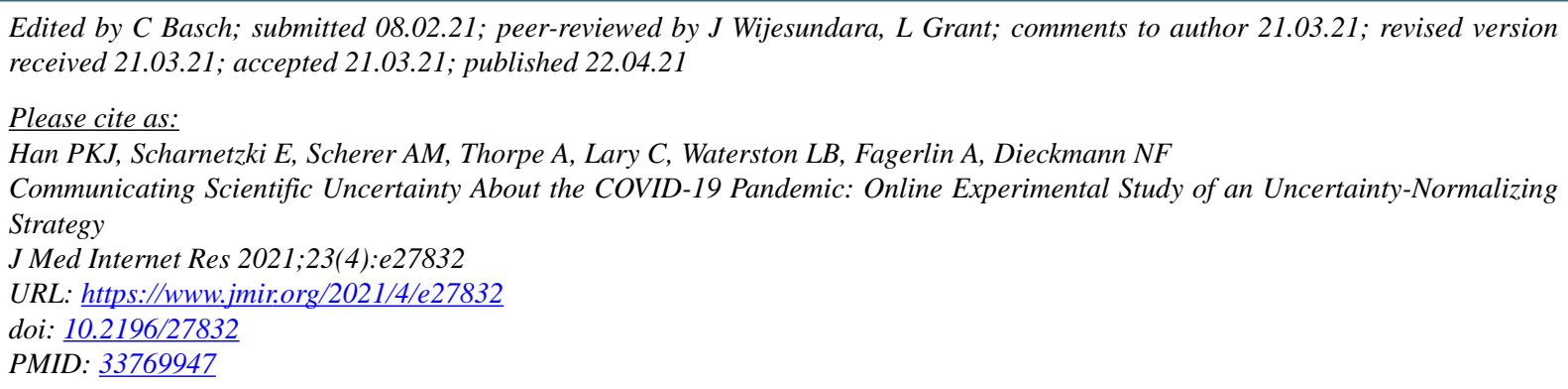

(CPaul K J Han, Elizabeth Scharnetzki, Aaron M Scherer, Alistair Thorpe, Christine Lary, Leo B Waterston, Angela Fagerlin, Nathan F Dieckmann. Originally published in the Journal of Medical Internet Research (https://www.jmir.org), 22.04.2021. This is an open-access article distributed under the terms of the Creative Commons Attribution License (https://creativecommons.org/licenses/by/4.0/), which permits unrestricted use, distribution, and reproduction in any medium, provided the original work, first published in the Journal of Medical Internet Research, is properly cited. The complete bibliographic information, a link to the original publication on https://www.jmir.org/, as well as this copyright and license information must be included. 